\title{
Up and Down the Black Hole Radio/X-Ray Correlation: The 2017 Mini-outbursts from Swift J1753.5-0127
}

\author{
R. M. Plotkin ${ }^{1}$ (1), J. Bright ${ }^{2}$, J. C. A. Miller-Jones ${ }^{1}$ (1), A. W. Shaw ${ }^{3}$, J. A. Tomsick ${ }^{4}$ (D), \\ T. D. Russell ${ }^{5}$, G.-B. Zhang ${ }^{6}$, D. M. Russell ${ }^{6}$, R. P. Fender ${ }^{2}$, J. Homan ${ }^{7,8}$ (i), P. Atri ${ }^{1}$, F. Bernardini ${ }^{6}$ (1) , J. D. Gelfand ${ }^{6}$ (1), \\ F. Lewis ${ }^{9,10}$, T. M. Cantwell ${ }^{11}$, S. H. Carey ${ }^{12}$, K. J. B. Grainge ${ }^{11}$ (D) J. Hickish $^{12,13}$, Y. C. Perrott ${ }^{12}$ (D) , N. Razavi-Ghods ${ }^{12}$, \\ A. M. M. Scaife ${ }^{11}$ (D) P. F. Scott ${ }^{12}$, and D. J. Titterington ${ }^{12}$ \\ ${ }^{1}$ International Centre for Radio Astronomy Research-Curtin University, GPO Box U1987, Perth, WA 6845, Australia; richard.plotkin@curtin.edu.au \\ ${ }^{2}$ Department of Physics, Astrophysics, University of Oxford, Keble Road, Oxford OX1 3RH, UK \\ ${ }^{3}$ Department of Physics, University of Alberta, 4-181 CCIS, Edmonton, AB T6G 2E1, Canada \\ ${ }^{4}$ Space Sciences Laboratory, 7 Gauss Way, University of California, Berkeley, CA 94720-7450, USA \\ 5 Anton Pannekoek Institute for Astronomy, University of Amsterdam, Science Park 904, NL-1098 XH Amsterdam, The Netherlands \\ ${ }^{6}$ New York University Abu Dhabi, P.O. Box 129188, Abu Dhabi, UAE \\ ${ }^{7}$ MIT Kavli Institute for Astrophysics and Space Research, 70 Vassar Street 37-582D, Cambridge, MA 02139, USA \\ ${ }^{8}$ SRON, Netherlands Institute for Space Research, Sorbonnelaan 2, 3584 CA Utrecht, The Netherlands \\ ${ }^{9}$ Faulkes Telescope Project, School of Physics \& Astronomy, Cardiff University, The Parade, CF24 3AA, Cardiff, Wales, UK \\ ${ }^{10}$ Astrophysics Research Institute, Liverpool John Moores University, 146 Brownlow Hill, Liverpool L3 5RF, UK \\ ${ }^{11}$ Jodrell Bank Centre for Astrophysics, Alan Turing Building, School of Physics and Astronomy, University of Manchester, M13 9PL, UK \\ ${ }^{12}$ Astrophysics Group, Cavendish Laboratory, 19 J. J. Thomson Avenue, Cambridge CB3 OHE, UK \\ ${ }^{3}$ Radio Astronomy Laboratory, University of California, Berkeley, CA 94720, USA \\ Received 2017 August 28; revised 2017 September 10; accepted 2017 September 14; published 2017 October 17
}

\begin{abstract}
The candidate black hole X-ray binary Swift J1753.5-0127 faded to quiescence in 2016 November after a prolonged outburst that was discovered in 2005. Nearly three months later, the system displayed renewed activity that lasted through 2017 July. Here, we present radio and X-ray monitoring over $\approx 3$ months of the renewed activity to study the coupling between the jet and the inner regions of the disk/jet system. Our observations cover low $\mathrm{X}$-ray luminosities that have not historically been well-sampled $\left(L_{\mathrm{X}} \approx 2 \times 10^{33}-10^{36} \mathrm{erg} \mathrm{s}^{-1} ; 1-10 \mathrm{keV}\right)$, including time periods when the system was both brightening and fading. At these low luminosities, Swift J1753.5 -0127 occupies a parameter space in the radio/X-ray luminosity plane that is comparable to "canonical" systems (e.g., GX 339-4), regardless of whether the system was brightening or fading, even though during its $\gtrsim 11$ year outburst, Swift J1753.5-0127 emitted less radio emission from its jet than expected. We discuss implications for the existence of a single radio/X-ray luminosity correlation for black hole X-ray binaries at the lowest luminosities $\left(L_{\mathrm{X}} \lesssim 10^{35} \mathrm{erg} \mathrm{s}^{-1}\right)$, and we compare to supermassive black holes. Our campaign includes the lowest luminosity quasi-simultaneous radio/X-ray detection to date for a black hole X-ray binary during its rise out of quiescence, thanks to early notification from optical monitoring combined with fast responses from sensitive multiwavelength facilities.
\end{abstract}

Key words: stars: black holes - stars: individual (Swift J1753.5-0127) - X-rays: binaries

\section{Introduction}

Black holes in X-ray binary systems (BHXBs) spend the majority of their time accreting relatively weakly, in a regime where a non-negligible fraction of their accretion power is channeled into compact relativistic jets (Fender et al. 2003; Körding et al. 2006). We define weakly accreting systems here as BHXBs with X-ray luminosities $L_{\mathrm{X}} \lesssim 10^{37} \mathrm{erg} \mathrm{s}^{-1}$, or similarly, Eddington ratios $L_{\mathrm{X}} / L_{\mathrm{Edd}} \lesssim 0.01,{ }^{14}$ which covers both the "hard" X-ray spectral state $\left(10^{-5} \lesssim L_{\mathrm{X}} / L_{\mathrm{Edd}} \lesssim 10^{-2}\right.$; Remillard \& McClintock 2006) and "quiescence" $\left(L_{\mathrm{X}} / L_{\mathrm{Edd}} \lesssim 10^{-5}\right.$; Plotkin et al. 2013). When weakly accreting BHXBs change their luminosities over day-to-week timescales, they trace out distinct paths through the radio luminosity $\left(L_{\mathrm{R}}\right)$ -X-ray luminosity $\left(L_{\mathrm{X}}\right)$ plane (e.g., Corbel et al. 2013a; Gallo et al. 2014). The radio emission is partially self-absorbed synchrotron radiation from a steady, unresolved, flat-spectrum jet (Blandford \& Königl 1979; Fender 2001), while the X-rays probe the inner regions of the accretion flow/jet. Thus, the

\footnotetext{
${ }^{14}$ The Eddington luminosity $L_{\mathrm{Edd}}=1.3 \times 10^{38}\left(M_{\mathrm{BH}} / M_{\odot}\right)$ erg s${ }^{-1}$, which we approximate as $L_{\text {Edd }} \sim 10^{39} \mathrm{erg} \mathrm{s}^{-1}$ here for a $\approx 10 M_{\odot}$ black hole.
}

presence of correlated radio and X-ray variability suggests a physical connection between the jet and the emission regions closest to the black hole (Heinz \& Sunyaev 2003; Markoff et al. 2003).

Three BHXB systems (GX 339-4, V404 Cygni, and XTE J1118+480) are known to display a nonlinear correlation of the form $L_{\mathrm{R}} \propto L_{\mathrm{X}}^{0.5-0.7}$ that extends unbroken over more than five orders of magnitude in $L_{\mathrm{X}}$ (Corbel et al. 2008, 2013a; Gallo et al. 2014), which we refer to as the "standard" radio/ $\mathrm{X}$-ray correlation. However, there is a population of "radiofaint" BHXBs at $L_{\mathrm{X}} \gtrsim 10^{36} \mathrm{erg} \mathrm{s}^{-1}\left(1^{-3} L_{\text {Edd }}\right)$ with radio luminosities that are 1-2 decades fainter than predicted by the "standard" correlation (e.g., Corbel et al. 2004; Cadolle Bel et al. 2007; Rodriguez et al. 2007; Xue \& Cui 2007; Soleri \& Fender 2011; Gallo et al. 2012), some of which show correlations as steep as $L_{\mathrm{R}} \propto L_{\mathrm{X}}^{1.4}$ (Coriat et al. 2011; Cao et al. 2014). Intriguingly, the "radio-faint" BHXB with the best radio/X-ray coverage, $\mathrm{H} 1743-322$, was unexpectedly observed to move horizontally across the $L_{R}-L_{X}$ plane when $L_{\mathrm{X}} \lesssim 4 \times 10^{36} \mathrm{erg} \mathrm{s}^{-1}$, until it rejoined the "standard" track around $L_{\mathrm{X}} \approx 10^{35} \mathrm{erg} \mathrm{s}^{-1}$ (Jonker et al. 2010; Coriat 
et al. 2011). Two other systems, MAXI J1659-152 and XTE J1752-223, appeared to also take similar paths between the two tracks (Jonker et al. 2012; Ratti et al. 2012).

The radio/X-ray luminosity plane becomes more poorly sampled as one moves toward lower luminosities (Miller-Jones et al. 2011). The BHXB candidate Swift J1753.5-0127 (hereafter J1753) recently afforded an opportunity to improve our coverage at low luminosities. J1753 was discovered in outburst in 2005 (Palmer et al. 2005), where it surprisingly remained for almost 12 years. The end of the outburst was noticed at optical wavelengths from 2016 September to November (Russell et al. 2016) by a program that regularly monitors $\sim 40$ BHXBs with the Faulkes Telescope Project (Lewis et al. 2008). J1753 then underwent a mini-outburst ${ }^{15}$ that was first detected in late January of 2017 and lasted through mid-April of 2017 (Al Qasim et al. 2017; Bright et al. 2017; Kong 2017; Shaw et al. 2017; Tomsick et al. 2017). In late April of 2017, approximately a week after J1753 returned below radio/X-ray detection thresholds, it underwent a second mini-outburst (Bernardini et al. 2017). J1753 returned to quiescence in the optical waveband by 2017 July (Zhang et al. 2017).

Here, we present results on the radio/X-ray luminosity correlation during the mini-outburst(s) of J1753, where we cover the radio/X-ray luminosity plane over the sparsely sampled $L_{\mathrm{X}} \approx 10^{33}-10^{36} \mathrm{erg} \mathrm{s}^{-1}$ regime. One particularly useful property of $\mathrm{J} 1753$ for studying BHXBs at low luminosities is that $\mathrm{J} 1753$ lies at high Galactic latitude $\left(b=12^{\circ}\right)$. Therefore, the line-of-sight absorption is relatively small $\left(N_{\mathrm{H}} \approx 2.0 \pm 0.3 \times\right.$ $10^{21} \mathrm{~cm}^{-2}$; Froning et al. 2014), allowing X-ray detections at lower luminosities than for most BHXBs. J1753 is also an intriguing target because it was established as a "radio-faint" BHXB (e.g., Cadolle Bel et al. 2007; Soleri et al. 2010) where it followed $L_{\mathrm{R}} \propto L_{\mathrm{X}}^{0.96}$ (Rushton et al. 2016) while in the hard state. J1753 is likely to host a black hole instead of a neutron star. For example, from the width of the (disk) $\mathrm{H} \alpha$ emission line in outburst, Shaw et al. (2016a) derived a compact object mass $\gtrsim 7 M_{\odot}$. Furthermore, during its outburst, the X-ray spectral and timing properties appeared more similar to other BHXBs than to neutron star X-ray binaries (e.g., Cadolle Bel et al. 2007; Durant et al. 2009; Soleri et al. 2010). The distance to J1753 is suggested to fall between 2 and $8 \mathrm{kpc}$ (Cadolle Bel et al. 2007; Froning et al. 2014). Following Rushton et al. (2016), we adopt $8 \mathrm{kpc}$ here, although adopting a lower value does not (qualitatively) alter our conclusions. Unless stated otherwise, we define X-ray luminosities from 1 to $10 \mathrm{keV}$, and we report uncertainties on radio and X-ray parameters at the $68 \%$ and $90 \%$ confidence levels, respectively.

\section{Observations}

Our data set combines observations from the Arcminute Microkelvin Imager Large Array (AMI-LA; AMI Consortium: Zwart et al. 2008; Hickish et al. 2017), the Karl G. Jansky Very Large Array (VLA), and the Very Long Baseline Array (VLBA) in the radio, and from the X-ray Telescope (XRT; Burrows et al. 2005) on board the Swift X-ray Mission

\footnotetext{
15 The optical flux peaked at a similar magnitude as before the initial descent into quiescence (see G.-B. Zhang et al. 2017, in preparation). Following Chen et al. (1997), we refer to such renewed activity as a mini-outburst. We also note that J1753 was too close to the Sun to observe from 2016 mid-November through 2017 mid-January in the optical and X-ray wavebands.
}

(Gehrels et al. 2004). Our analysis is described below and summarized in Table 1.

\subsection{AMI-LA}

AMI-LA monitored J1753 starting on 2017 February 15, observing a total of 35 times over $\sim 100$ days (although the last AMI-LA detection was on 2017 April 8, about midway through our campaign). Observations generally lasted 3-4 hr, with typical image noises $\sigma_{\mathrm{rms}} \approx 0.04 \mathrm{mJy}$ beam $^{-1}$. Observations were carried out at a central frequency of $15.5 \mathrm{GHz}$ with a total bandwidth of $5 \mathrm{GHz}$. We observed the calibrator source $\mathrm{J} 1804+0101$ for $\sim 2$ minutes for every 9 minutes on source to find the complex gain solutions. Data were binned into eight channels, each with a width of $625 \mathrm{MHz}$, and the data were calibrated and flagged for radio frequency interference (RFI) with the AMI reduction pipeline REDUCE_DC. Further RFI flagging was performed in the Common Astronomy Software Application CASA v4.2.2 (McMullin et al. 2007), and imaging was performed with the task CLEAN, setting a halting threshold of $3 \sigma_{\text {rms }}$. To extract flux measurements, we used the PYTHON-based source extractor PYSE, which was developed as part of the LOFAR Transient Pipeline (TRAP; Swinbank et al. 2015). A two-dimensional Gaussian with the same dimensions as the synthesized beam was used to fit sources in the image plane. We detected an unresolved source consistent with the location of J1753 in eight of our observations, using a detection threshold of $3.5 \sigma_{\mathrm{rms}}$ and including all pixels with values $>3 \sigma_{\mathrm{rms}}$ during the fitting analysis, where $\sigma_{\text {rms }}$ is the statistical error (the error bars reported in Table 1 also include a $10 \%$ systematic error from uncertainties on the flux density calibration scale). Throughout this paper, we only consider these eight detections and ignore upper limits, as we initiated our (more sensitive) VLA observations shortly after J1753 was no longer detected by AMI-LA.

\subsection{VLA}

We observed J1753 with the VLA after its initial descent into quiescence on 2016 November 5 and 7 (project code VLA/16A-060, see Plotkin et al. 2016), and we also obtained three epochs during the mini-outbursts on 2017 April 19, 21, and 29 (project code VLA/17A-430, awarded through Director's Discretionary Time). The observational setups were similar for all observations, except that the VLA was in the most extended (A) configuration during the 2016 November observations, and it was in the most compact (D) configuration during the observations from 2017.

We used two basebands centered at 9.0 and $10.65 \mathrm{GHz}$, with 1.9 and $1.8 \mathrm{GHz}$ bandwidth respectively. Observations lasted for $1 \mathrm{hr}$ each ( $\approx 32-38$ minutes on source), except for 2016 November 7 , which lasted for $2.25 \mathrm{hr}(\approx 105 \mathrm{~min}$ on source). We observed the phase calibrator J1743-0350 every 5-8 minutes to solve for the complex gains, and we set the flux amplitude scale using 3C 286 on 2016 November 5, 7 and 2017 April 19, and 3C 48 on the other two epochs. Weather conditions were poor on 2016 November 5, and we could not obtain useful phase solutions to calibrate the data. Weather was good during the other four epochs.

Data were processed using standard procedures in CASA v 4.7.1, and the flux scale was set using the task SETJY and the Perley \& Butler (2013) coefficients. We imaged the field with the task CLEAN, using two Taylor terms to model the frequency dependence of other sources in the field, and Briggs weighting with robust $=1$ to reduce sidelobes from other sources in the 
Table 1

Summary of Radio and X-Ray Observations

\begin{tabular}{|c|c|c|c|c|c|c|c|}
\hline (1) & (2) & (3) & $\begin{array}{c}f_{r} \\
\left(\mathrm{mJy} \mathrm{bm}^{-1}\right) \\
(4)\end{array}$ & $\begin{array}{c}\log \left(\nu L_{\nu}\right)_{5 \mathrm{GHz}} \\
\left(\mathrm{erg} \mathrm{s}^{-1}\right) \\
(5)\end{array}$ & $\begin{array}{l}\Gamma \\
(6)\end{array}$ & $\begin{array}{c}f_{0.6-10 \mathrm{keV}} \\
\left(10^{-12} \mathrm{erg} \mathrm{s}^{-1} \mathrm{~cm}^{-2}\right) \\
(7)\end{array}$ & $\begin{array}{c}\log L_{1-10 \mathrm{keV}} \\
\left(\mathrm{erg} \mathrm{s}^{-1}\right) \\
(8)\end{array}$ \\
\hline 2016 Nov $6^{\mathrm{a}}$ & $\mathrm{XRT} / \mathrm{PC}$ & 57698.08592 & $\ldots$ & $\ldots$ & $1.7^{\mathrm{b}}$ & $<0.3$ & $<33.3$ \\
\hline $2016 \operatorname{Nov} 7^{\mathrm{a}}$ & $\mathrm{XRT} / \mathrm{PC}$ & 57699.48564 & $\ldots$ & $\ldots$ & $1.7^{\mathrm{b}}$ & $<0.2$ & $<33.0$ \\
\hline $2016 \operatorname{Nov} 7^{c}$ & VLA & 57699.91875 & $<0.008$ & $<27.5$ & $\ldots$ & $\ldots$ & $\ldots$ \\
\hline 2017 Feb 15 & AMI-LA & 57799.23803 & $0.291 \pm 0.055$ & $29.0 \pm 0.1$ & $\ldots$ & $\ldots$ & $\ldots$ \\
\hline 2017 Feb 16 & $\mathrm{XRT} / \mathrm{PC}$ & 57800.07281 & $\ldots$ & $\ldots$ & $1.8 \pm 0.2$ & $61.8_{-19.5}^{+29.6}$ & $35.6_{-0.1}^{+0.2}$ \\
\hline 2017 Feb 19 & AMI-LA & 57803.25274 & $0.346 \pm 0.048$ & $29.1 \pm 0.1$ & $\ldots$ & $\ldots$ & $\ldots$ \\
\hline 2017 Feb 19 & $\mathrm{XRT} / \mathrm{PC}$ & 57803.33760 & $\ldots$ & $\ldots$ & $1.9 \pm 0.2$ & $71.4_{-23.5}^{+45.8}$ & $35.7_{-0.1}^{+0.3}$ \\
\hline 2017 Feb 22 & $\mathrm{XRT} / \mathrm{PC}$ & 57806.39433 & $\cdots$ & $\cdots$ & $1.6 \pm 0.1$ & $81.7_{-13.8}^{+22.0}$ & $35.7_{-0.1}^{+0.1}$ \\
\hline 2017 Feb 23 & XRT/WT & 57807.91082 & $\ldots$ & $\ldots$ & $1.8 \pm 0.1$ & $82.7_{-13.8}^{+23.2}$ & $35.7_{-0.1}^{+0.1}$ \\
\hline 2017 Feb 24 & XRT/WT & 57808.91153 & $\cdots$ & $\cdots$ & $1.8 \pm 0.2$ & $98.9_{-23.3}^{+36.0}$ & $35.8_{-0.1}^{+0.2}$ \\
\hline 2017 Feb 25 & XRT/PC & 57809.31692 & $\ldots$ & $\ldots$ & $1.4 \pm 0.2$ & $97.3_{-33.2}^{+60.4}$ & $35.8_{-0.1}^{+0.3}$ \\
\hline 2017 Mar 14 & AMI-LA & 57826.23219 & $0.223 \pm 0.048$ & $28.9 \pm 0.1$ & $\ldots$ & $\ldots$ & $\ldots$ \\
\hline 2017 Mar 22 & AMI-LA & 57834.13557 & $0.283 \pm 0.051$ & $29.0 \pm 0.1$ & $\ldots$ & $\ldots$ & $\ldots$ \\
\hline 2017 Mar 25 & AMI-LA & 57837.11836 & $0.173 \pm 0.050$ & $28.8 \pm 0.1$ & $\ldots$ & $\ldots$ & $\ldots$ \\
\hline 2017 Mar 25 & XRT/WT & 57837.61163 & $\ldots$ & $\ldots$ & $1.7 \pm 0.2$ & $38.6_{-8.6}^{+25.2}$ & $35.4_{-0.1}^{+0.3}$ \\
\hline 2017 Mar 27 & AMI-LA & 57839.10113 & $0.199 \pm 0.044$ & $28.9 \pm 0.1$ & $\ldots$ & .. & $\ldots$ \\
\hline 2017 Apr 1 & XRT/WT & 57844.01194 & $\ldots$ & $\ldots$ & $2.2 \pm 0.3$ & $48.6_{-31.2}^{+102.6}$ & $35.5_{-0.3}^{+0.9}$ \\
\hline 2017 Apr 1 & AMI-LA & 57844.09925 & $0.201 \pm 0.044$ & $28.9 \pm 0.1$ & $\ldots$ & $\ldots$ & $\ldots$ \\
\hline 2017 Apr 6 & XRT/WT & 57849.58618 & $\ldots$ & $\ldots$ & $1.8 \pm 0.3$ & $22.4_{-6.8}^{+16.7}$ & $35.2_{-0.1}^{+0.3}$ \\
\hline 2017 Apr 8 & AMI-LA & 57851.10923 & $0.202 \pm 0.056$ & $28.9 \pm 0.1$ & $\ldots$ & $\ldots$ & $\ldots$ \\
\hline 2017 Apr 8 & XRT/WT & 57851.24913 & $\ldots$ & $\ldots$ & $2.3 \pm 0.4$ & $23.1_{-7.4}^{+17.8}$ & $35.1_{-0.1}^{+0.3}$ \\
\hline 2017 Apr 13 & VLBA & 57856.54167 & $<0.160$ & $<28.8$ & $\ldots$ & $\ldots$ & $\ldots$ \\
\hline 2017 Apr 15 & $\mathrm{XRT} / \mathrm{PC}$ & 57858.01942 & $\ldots$ & $\ldots$ & $1.8 \pm 0.6$ & $4.9_{-2.3}^{+8.5}$ & $34.5_{-0.2}^{+0.7}$ \\
\hline 2017 Apr 18 & $\mathrm{XRT} / \mathrm{PC}$ & 57861.87166 & $\ldots$ & $\ldots$ & $1.5 \pm 0.5$ & $1.9_{-1.4}^{+2.4}$ & $34.1_{-0.3}^{+0.5}$ \\
\hline 2017 Apr 19 & VLA & 57862.30750 & $0.045 \pm 0.007$ & $28.2 \pm 0.1$ & $\ldots$ & $\ldots$ & $\ldots$ \\
\hline 2017 Apr 20 & XRT/WT & 57863.98851 & $\ldots$ & $\ldots$ & $1.7^{\mathrm{b}}$ & $<2.0$ & $<34.1$ \\
\hline 2017 Apr 21 & VLA & 57864.60620 & $<0.016$ & $<27.8$ & $\cdots$ & $\cdots$ & $\cdots$ \\
\hline 2017 Apr 22 & $\mathrm{XRT} / \mathrm{PC}$ & 57865.91086 & $\ldots$ & $\ldots$ & $1.7^{\mathrm{b}}$ & $<0.2$ & $<33.1$ \\
\hline 2017 Apr 29 & VLA & 57872.59650 & $0.019 \pm 0.005$ & $27.9 \pm 0.1$ & $\ldots$ & $\ldots$ & $\ldots$ \\
\hline 2017 Apr 29 & $\mathrm{XRT} / \mathrm{PC}$ & 57872.77287 & $\ldots$ & $\ldots$ & $1.7^{\mathrm{b}}$ & $0.2_{-0.2}^{+0.4}$ & $33.2_{-0.3}^{+0.8}$ \\
\hline 2017 May 6 & $\mathrm{XRT} / \mathrm{PC}$ & 57879.67537 & $\ldots$ & $\ldots$ & $1.8 \pm 0.4$ & $2.4_{-1.3}^{+4.4}$ & $34.2_{-0.2}^{+0.8}$ \\
\hline 2017 May 15 & $\mathrm{XRT} / \mathrm{PC}$ & 57888.43380 & $\ldots$ & $\ldots$ & $1.7^{\mathrm{b}}$ & $0.2_{-0.2}^{+0.4}$ & $33.2_{-0.3}^{+0.8}$ \\
\hline
\end{tabular}

Notes. Column (1) calendar date of each observation. Column (2) the telescope used for each observation. For Swift/XRT, we specify if the observations were taken in photon counting (PC) or window timing (WT) mode. Column (3) modified Julian date of each observation. Column (4) peak radio flux density at the central observing frequency (15.5 GHz for AMI-LA, 9.8 GHz for the VLA, and $4.98 \mathrm{GHz}$ for the VLBA). All radio error bars are reported at the $68 \%$ confidence level (and they include systematic errors on the flux density calibration scale) and upper limits are at the $3 \sigma_{\text {rms }}$ level for the VLA and $5 \sigma_{\text {rms }}$ for the VLBA. Column (5) logarithm of the radio luminosity at $5 \mathrm{GHz}$, assuming a flat radio spectrum (see Section 3.2) and $d=8 \mathrm{kpc}$. Column (6) best-fit photon index $\Gamma$ from each $S w i f t$ observation. All X-ray error bars are reported at the $90 \%$ confidence level. Column (7) model X-ray flux over the 0.6-10 keV Swift/XRT energy band. Upper limits are at the 99\% confidence level. Column (8) logarithm of the $1-10 \mathrm{keV}$ X-ray luminosity, assuming $d=8 \mathrm{kpc}$.

${ }^{a}$ Observation first reported by Shaw et al. (2016b).

${ }^{\mathrm{b}}$ Due to a low number of photons, X-ray fluxes were estimated using an absorbed power-law model with photon index $\Gamma=1.7$ and a column density $N_{\mathrm{H}}=2 \times 10^{21} \mathrm{~cm}^{-2}$

${ }^{\mathrm{c}}$ Observation first reported by Plotkin et al. (2016).

field. J1753 was detected on 2017 April 19 and 29, with peak flux densities of $45\left(6.1 \sigma_{\text {rms }}\right)$ and $19\left(3.6 \sigma_{\mathrm{rms}}\right) \mu \mathrm{Jy} \mathrm{bm}^{-1}$ at $9.8 \mathrm{GHz}$, respectively, measured with the task IMFIT by fitting a point source model in the image plane. No radio emission was detected from J1753 on 2016 November 7 or on 2017 April 21, and we derived $3 \sigma_{\text {rms }}$ upper limits of $f_{\nu}<8 \mu \mathrm{Jy} \mathrm{bm}^{-1}$ and $f_{\nu}<16 \mu \mathrm{Jy} \mathrm{bm}^{-1}$, respectively. Error bars on the VLA flux densities in Table 1 include statistical errors and 5\% systematic errors (the latter is the accuracy on the VLA flux density calibration scale). ${ }^{16}$

\footnotetext{
$\overline{16}$ https://science.nrao.edu/facilities/vla/docs/manuals/oss/performance/ fdscale
}

\section{3. $V L B A$}

We observed J1753 with the VLBA on 2017 April 13 (10:30-15:30 UT), as part of a filler-time astrometric program (project code BM449). We observed at a central frequency of $4.98 \mathrm{GHz}$, with an observing bandwidth of $256 \mathrm{MHz}$. Two 30-minute periods, one at the start of the observation and one at the end, were dedicated to a geodetic block, observing a range of bright calibrators across the sky to correct for unmodeled tropospheric and clock errors in the correlated data. For the remaining four hours, we switched between phase reference calibrator sources, J1753, and an astrometric check source. We used the nearby compact 
source $\mathrm{J} 1752-0147$ (R.A. $=17^{\mathrm{h}} 52^{\mathrm{m}} 18 \mathrm{~s} 3637813, \quad$ decl. $=$ $-01^{\circ} 47^{\prime} 16$ ". 685462 (J2000); only 27 arcmin from J1753) as our primary phase reference calibrator, using a 3-minute cycle time (110 s on target, $70 \mathrm{~s}$ on calibrator), and we observed the brighter but more distant calibrator J1743-0350 every 20 minutes to calibrate the delays and rates. The data were calibrated according to standard procedures within the Astronomical Image Processing System (AIPS; Greisen 2003). No radio source was detected above a $5 \sigma_{\text {rms }}$ upper limit of $0.16 \mathrm{mJy} \mathrm{bm}^{-1}$ (the systematic uncertainty on the amplitude calibration is $\approx 5 \%$ ).

\subsection{Swift XRT}

X-ray observations were taken with Swift/XRT shortly after the initial outburst decay (on 2016 November 6 and 7; see Shaw et al. (2016b) for details), and during the mini-outbursts from 2017 February 16 to 2017 May 15 (Target ID: 00030090). Swift/XRT observed the source in auto-exposure mode for the majority of the observations, adjusting the $\mathrm{CCD}$ readout mode between windowed timing (WT) and photon counting (PC) according to the observed count rate.

Data were reprocessed using the HEASOFT v6.19 17 task XRTPIPELINE. WT count rates were extracted using a circular region 20 pixels in radius $\left(\approx 47^{\prime \prime}\right)$. Background count rates in WT mode were extracted from an annulus centered on the source with inner and outer radii of 80 and 120 pixels, respectively. PC mode source count rates were extracted from a circular region of the same radius as in WT mode, and the average count rate was then calculated in order to determine if photon pile-up was significant. PC observations with count rates higher than 0.5 counts $\mathrm{s}^{-1}$ were re-extracted using an annulus with a 20-pixel outer radius and the central portion of the point-spread function excluded. The radius of the excluded region was determined using NASA's XIMAGE package ${ }^{18}$ and ranged from $\sim 2$ to 4 pixels. PC mode background count rates were extracted from an annulus centered on the source with inner and outer radii of 50 and 70 pixels, respectively. The number of (net) source counts ranged from $\approx 10$ to 2500 counts.

Spectra of each observation were extracted and spectral fits were performed in XSPEC v12.9.0 (Arnaud et al. 1996). Due to the small number of counts in some observations ( 8 epochs have $<100$ net counts), we grouped each spectrum to have a minimum of one count per energy bin, and we performed the spectral fitting using Cash statistics for background subtracted spectra (W-statistics; Cash 1979). Interstellar absorption was accounted for by the TBABS model with Wilms et al. (2000) abundances and Verner et al. (1996) photoionization crosssections. We obtained adequate fits to all spectra using an absorbed power-law model (POWERLAW; no model fit was improved by adding a DISKBB component). We then extracted unabsorbed model fluxes and $90 \%$ error bars in the $0.6-10 \mathrm{keV}$ and the $1-10 \mathrm{keV}$ bands with the tool CFLUX (the error bars also incorporate uncertainties related to the best-fit model parameters).

For observations without enough X-ray counts to fit a spectral model ( $\lesssim 30-50$ counts), we required detections to be significant at the $>99 \%$ confidence level, according to Poisson statistics in the presence of background (Kraft et al. 1991). For

\footnotetext{
17 https://heasarc.nasa.gov/lheasoft/

18 http://www.swift.ac.uk/analysis/xrt/pileup.php
}
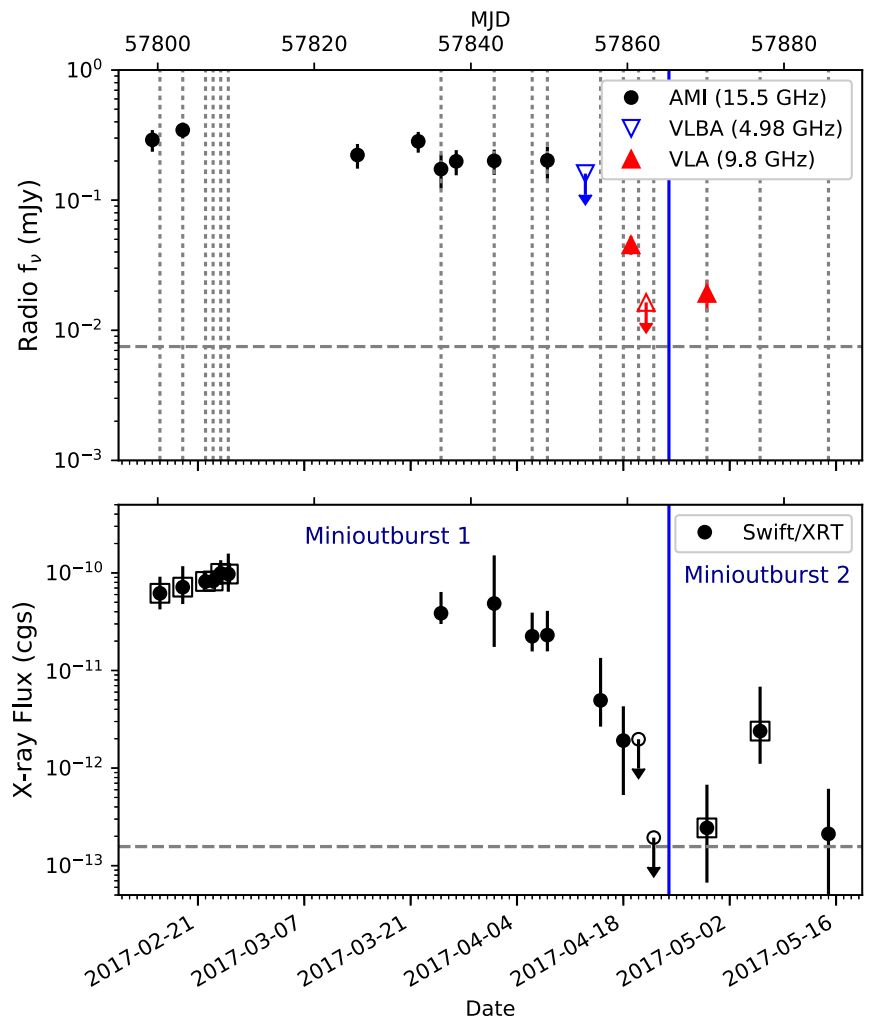

Figure 1. Radio and X-ray light curves of the 2017 mini-outburst(s). The top panel shows radio flux densities from AMI-LA (15.5 GHz; black circles), the VLBA (4.98 GHz; blue upside down triangle), and the VLA $(9.8 \mathrm{GHz}$; red triangles), with error bars representing $68 \%$ confidence. The vertical dotted lines mark the epochs of our X-ray observations. The bottom panel shows $\mathrm{X}$-ray fluxes (in units of $\mathrm{erg} \mathrm{s}^{-1} \mathrm{~cm}^{-2}$ ) from 0.6 to $10 \mathrm{keV}$ with errors illustrated at $90 \%$ confidence. The vertical blue solid line illustrates the approximate boundary between the two mini-outbursts, and the data points circumscribed by squares represent when $\mathrm{J} 1753$ was rising out of quiescence. The horizontal dashed lines show the deepest radio and X-ray flux limits yet for J1753 in quiescence, both obtained on 2016 November 7.

these low-count observations, we assumed a power-law model with $\Gamma=1.7$ and $N_{\mathrm{H}}=2 \times 10^{21} \mathrm{~cm}^{-2}$ (Froning et al. 2014) to estimate a flux. For error bars, we adopted $90 \%$ confidence intervals from Kraft et al. (1991), and we factored in a photon index that was allowed vary from $1<\Gamma<2.5$.

\section{Results}

\subsection{Light Curves}

Radio and X-ray light curves are displayed in Figure 1, which span from February through May of 2017. J1753 dropped below our radio and X-ray detection thresholds from April 20 to 22 (despite being detected at both wavebands two days earlier). J1753 was subsequently detected at both wavebands again on April 29, implying a second mini-outburst. During the rise of the second mini-outburst, we caught J1753 shortly after it brightened above our detection thresholds.

\subsection{Radio/X-Ray Correlation}

A total of eight of our radio (AMI-LA/VLA) and X-ray observations were taken $<1$ day apart, which we place on the radio/X-ray luminosity plane in Figure 2. Our VLBA radio limit from 2017 April 13 was taken 1.5 days before an X-ray observation. To place that epoch on the radio/X-ray plane, we 


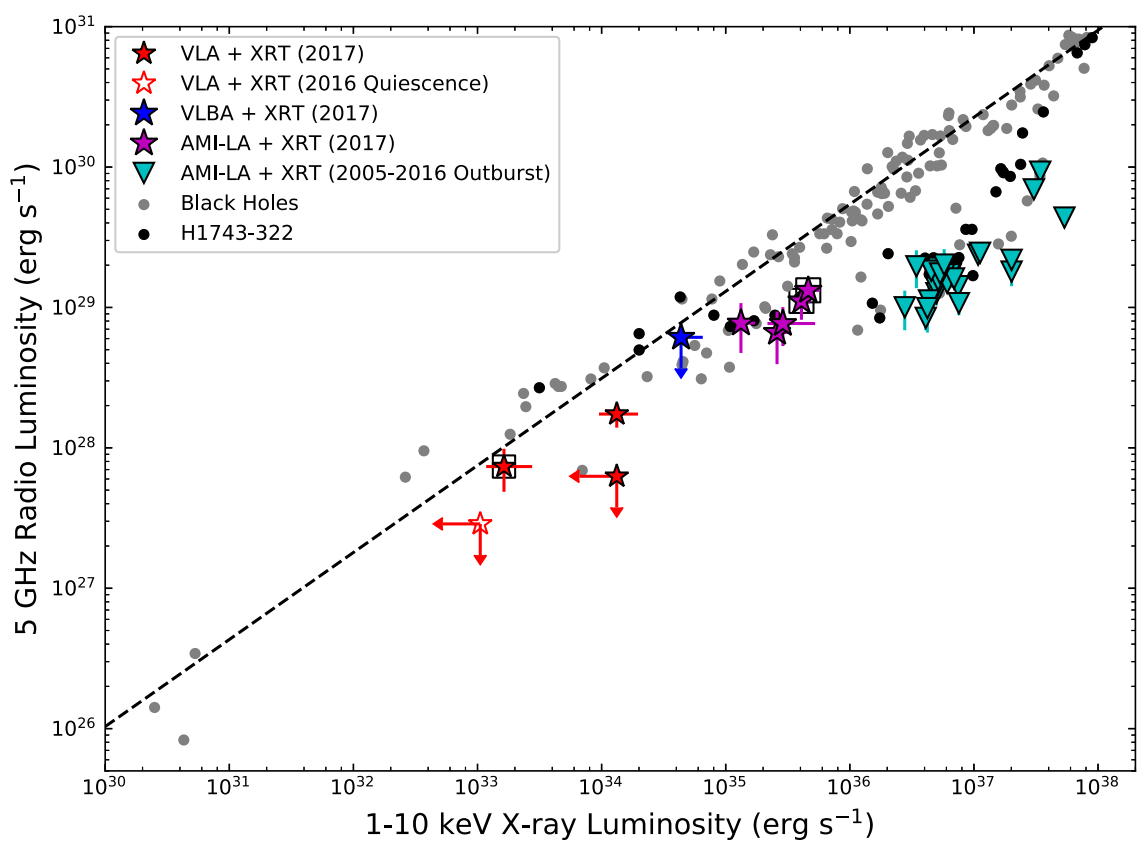

Figure 2. J1753 on the radio/X-ray luminosity plane, assuming it is located $8 \mathrm{kpc}$ away. The red star symbols show epochs with the VLA (the open symbol represents our deeper limit in quiescence from 2016 November), the blue star symbol with the VLBA, and the purple star symbols with AMI-LA. Data points during the rise out of quiescence (three epochs) are circumscribed by squares. The cyan upside down triangles show the location of J1753 in the "radio-faint" hard state during its $\gtrsim 11$ year outburst (data taken from Rushton et al. 2016). For uniformity, all X-ray error bars are rescaled to 68\% confidence. A sample of comparison BHXBs are shown as gray circles, with H1743-322 highlighted with dark circles. The dashed line shows the radio/X-ray correlation of GX 339-4 from Gallo et al. (2014), to illustrate the "standard" track (see Section 3.2). J1753 lies close to the "standard" track at low luminosities $L_{\mathrm{X}} \lesssim 10^{36} \mathrm{erg} \mathrm{s}^{-1}$.

interpolate the X-ray light curve between 2017 April 8 and 18 to the time of the VLBA observation (the X-ray light curve appears to be exponentially decaying during that time period; see Figure 1). We attempt to improve the "simultaneity" of the other eight epochs by interpolating the radio and X-ray light curves, but doing so does not alter any results (within errors). Our observations include epochs when the X-ray flux is rising (three data points circumscribed by squares in Figure 2) and others when $\mathrm{J} 1753$ is fading back into quiescence, providing a rare opportunity to compare disk/jet couplings in both directions.

For ease of comparison to the literature, we extrapolate all radio observations to $5 \mathrm{GHz}$, assuming a flat radio spectrum. The assumption of a flat radio spectrum appears reasonable from Cadolle Bel et al. (2007), who measured $\alpha_{r}=0.03 \pm 0.03\left(f_{\nu} \propto \nu^{\alpha_{r}}\right)$ for $\mathbf{J} 1753$ from radio observations in 2005. However, Tomsick et al. (2015) measured an inverted radio spectrum from observations taken in 2014 $\left(\alpha_{\mathrm{r}}=0.29 \pm 0.05\right)$. We therefore add uncertainties to the radio luminosity error bars in Figure 2 to account for a radio spectrum that could be as inverted as $\alpha_{\mathrm{r}}=0.3$.

For comparison, we also display radio and X-ray observations of J1753 during its 2005-2016 outburst in Figure 2, when $\mathrm{J} 1753$ was in the radio-faint hard state (Rushton et al. 2016). To illustrate the "standard" track in Figure 2, we adopt the best-fit to the BHXB GX 339-4 from Gallo et al. (2014; we use GX $339-4$ as a representative example because it has the most data coverage for any "standard" track BHXB, taken over multiple outbursts; Corbel et al. 2013a). We also highlight the path H1743-322 took through the $L_{\mathrm{R}}-L_{\mathrm{X}}$ plane to emphasize that J1753 appears to occupy a similar parameter space.

\section{Discussion}

Figure 2 shows that during the 2017 mini-outbursts, J1753 occupies a region of the $L_{\mathrm{R}}-L_{\mathrm{X}}$ plane that is inconsistent with the $L_{\mathrm{R}} \propto L_{\mathrm{X}}^{0.96}$ correlation it followed during its outburst (Rushton et al. 2016). Even though J1753 appears to always fall below the radio/X-ray correlation defined by GX 339-4 (dashed solid line), its path through the radio/X-ray plane is clearly different above and below $L_{\mathrm{X}} \approx 10^{36} \mathrm{erg} \mathrm{s}^{-1}$ (i.e., J1753 does not simply follow a single, lower-normalization correlation that is parallel to the "standard track"). However, it is unclear whether the difference above and below $10^{36} \mathrm{erg} \mathrm{s}^{-1}$ is driven by the X-ray luminosity, by the Eddington ratio, or by other details related to the physics of the mini-outbursts.

Intriguingly, J1753 occupies a similar parameter space in Figure 2 as H1743-322 (black circles), a BHXB that was observed to move horizontally across the $L_{\mathrm{R}}-L_{\mathrm{X}}$ plane as it transitioned from the hard state to quiescence at the end of an outburst (Jonker et al. 2010; Coriat et al. 2011). It very likely could be the case that $\mathrm{J} 1753$ also moved horizontally across the $L_{\mathrm{R}}-L_{\mathrm{X}}$ plane. However, because the initial decay was not monitored in the radio and X-ray in 2016, we cannot exclude a scenario where J1753 faded down its "radio-faint" hard state $L_{\mathrm{R}} \propto L_{\mathrm{X}}^{0.96}$ correlation during the initial decay, and then it rose and faded along a path close to the "standard" track during the mini-outbursts. Unfortunately, J1753 did not reach high enough X-ray luminosities during the mini-outbursts to determine if it would have moved horizontally back to the "radio-faint" hard state. Regardless, our campaign reinforces the notion that "radio-faint" BHXBs can follow paths similar to the "standard" track at the lowest X-ray luminosities, and we 
still lack observational evidence for the existence of a "radiofaint" BHXB branch below $L_{\mathrm{X}} \approx 10^{36} \mathrm{erg} \mathrm{s}^{-1}$.

We note that while J1753 is illustrated in Figure 2 assuming a distance of $8 \mathrm{kpc}$, it has also been suggested that the source distance could be as low as 2-4 kpc (e.g., Cadolle Bel et al. 2007; Froning et al. 2014). Adopting a lower distance would of course not change our primary conclusion that J1753 appears to follow different radio/X-ray correlations at high and low luminosities. However, if J1753 were to be closer than $8 \mathrm{kpc}$, then it would fall even farther below the "standard" track at low luminosities, and it would not occupy precisely the same parameter space as $\mathrm{H} 1743-322$. The better agreement with other BHXBs on $L_{\mathrm{R}}-L_{\mathrm{X}}$ at $8 \mathrm{kpc}$ might suggest that $\mathrm{J} 1753$ indeed lies at a larger distance. The distance estimate will hopefully be improved in the future through studies on the quiescent optical counterpart.

\subsection{The Radio/X-Ray Correlation in Quiescence}

As BHXBs fade toward quiescence, they may enter a jetdominated state, where a substantial fraction of the accretion power could be channeled into the jet as mechanical power instead of being liberated as X-rays from within the accretion flow (Fender et al. 2003). ${ }^{19}$ Yuan \& Cui (2005) predict that at luminosities below $L_{\mathrm{X}} \approx 10^{33}-10^{34} \mathrm{erg} \mathrm{s}^{-1}\left(10^{-6}-10^{-5} L_{\mathrm{Edd}}\right)$, the jet may also dominate the radiative output with the observed $\mathrm{X}$-ray emission arising predominantly from non-thermal emission from a synchrotron cooled jet. As a consequence, Yuan \& Cui (2005) predict that the radio/X-ray luminosity correlation will follow a steeper slope in quiescence. Our campaign on J1753 detected radio and X-ray emission near the "standard" track at a luminosity as low as $L_{\mathrm{X}} \approx 2 \times 10^{33} \mathrm{erg} \mathrm{s}^{-1}$ $\left(\approx 10^{-5.7} L_{\mathrm{Edd}}\right)$, implying that if the "standard" track steepens, then it must do so at an even lower luminosity. Plotkin et al. (2017) more rigorously showed for the BHXB V404 Cygni that the "standard" radio/X-ray correlation maintains its slope to at least $L_{\mathrm{X}} \approx 3 \times 10^{32} \mathrm{erg} \mathrm{s}^{-1}\left(\approx 10^{-6.5} L_{\mathrm{Edd}}\right)$ in that source. Furthermore, radio detections of the BHXBs A 0620-00, MWC 656, and XTE J1118+480 all fall on an extrapolation of the "standard" track to $L_{\mathrm{X}} \approx 2 \times 10^{30}-1 \times 10^{31} \mathrm{erg} \mathrm{s}^{-1}$ $\left(\approx 10^{-8.7}-10^{-8.0} L_{\text {Edd }}\right.$; Gallo et al. 2006, 2014; Ribó et al. 2017). From the above, it seems reasonable to exclude the possibility that all BHXBs follow a steeper radio/X-ray correlation at the lowest luminosities.

By incorporating a mass normalization term, the "standard" radio/X-ray correlation can be extended to include supermassive black holes that power low-luminosity active galactic nuclei (LLAGN), i.e., the fundamental plane of black hole activity (Merloni et al. 2003; Falcke et al. 2004). Intriguingly, Xie \& Yuan (2017) find a steepening of the slope of the fundamental plane for quiescent LLAGN at $L_{\mathrm{X}} \lesssim 10^{-6} L_{\mathrm{Edd}}$ (also see Yuan et al. 2009), in line with the predictions of Yuan \& Cui (2005; although see Dong \& Wu 2015 for an alternative view). There is thus some tension toward understanding why a steeper correlation may exist for quiescent LLAGN, while observations of BHXBs so far do not show any evidence for a steepening. Whether or not there is a single "track" in quiescence will have consequences not just on our understanding of quiescent accretion and jet physics, but also on our ability to use the fundamental plane to search for new

\footnotetext{
19 Some of the accretion power can also be advected through the black hole event horizon (e.g., Garcia et al. 2001).
}

populations of quiescent BHXBs (e.g., Maccarone 2005; Strader et al. 2012; Chomiuk et al. 2013; Fender et al. 2013; Miller-Jones et al. 2015; Tetarenko et al. 2016) and LLAGN, particularly in the intermediate mass range (e.g., Miller-Jones et al. 2012; Cseh et al. 2015; Koliopanos et al. 2017; Mezcua 2017).

One explanation for the apparent difference in the radio/ X-ray correlation slope between quiescent BHXBs and LLAGN is that we simply have not yet observed enough BHXBs to detect a (sub)population that proceeds down a steeper track in quiescence. It is also possible that for LLAGN, an extra source of X-ray emission could be contributed by $\mathrm{X}$-ray binaries near the nucleus of the host galaxy (e.g., Miller et al. 2015), which could artificially steepen the radio/X-ray correlation slope (and add additional scatter). A third explanation, which we explore in more detail below, is that BHXBs do not have black holes that are massive enough for a synchrotron cooled jet to ever dominate the X-ray waveband.

For a non-thermal distribution of synchrotron emitting particles accelerated along a jet, the frequency above which particles suffer synchrotron radiative cooling losses scales as $\nu_{c} \propto \dot{m}^{-3 / 2} M_{\mathrm{BH}}^{-1 / 2}$ (Heinz 2004), where $\dot{m}$ is the Eddington normalized mass accretion rate $\left(\dot{M} / \dot{M}_{\text {Edd }}\right){ }^{20}$ Thus, scaling from stellar mass $\left(\approx 10 M_{\odot}\right)$ to supermassive scales $\left(\approx 10^{6}-10^{9} M_{\odot}\right)$ would lower the frequency of the synchrotron cooling break by $\approx 2.5-4$ decades in frequency. It could therefore be possible for synchrotron cooled radiation to appear in the X-ray waveband for supermassive black holes but not for BHXBs, purely from mass-scaling arguments that are independent of accretion rate (see Plotkin et al. 2012 for observations supporting this interpretation). If this is correct, then jet emission can only dominate the X-ray spectrum of quiescent BHXBs if it is synchrotron self-Compton, and/or if the jet is not radiatively cooled $^{21}$ (Gallo et al. 2007; Corbel et al. 2008; Plotkin et al. 2015, 2017; Connors et al. 2017); otherwise, the radiatively inefficient accretion flow will always dominate the X-ray spectrum of quiescent BHXBs (e.g., Esin et al. 1997; McClintock et al. 2003; Zdziarski et al. 2004; Sobolewska et al. 2011; Qiao \& Liu 2013; Yuan \& Narayan 2014).

\subsection{Comparing the Rise and Decay Out of Quiescence}

Radio and X-ray detections of a BHXB during the rise out of quiescence are rare at low luminosities, and our radio/X-ray detections of J1753 at $\left(L_{\mathrm{X}}, L_{\mathrm{R}}\right) \approx\left(2 \times 10^{33}, 8 \times 10^{27}\right) \mathrm{erg} \mathrm{s}^{-1}$ represent the lowest quasi-simultaneous luminosity detections yet in the rising hard state (for this data point, the radio/X-ray observations were only separated by $4.2 \mathrm{hr}$ ). Even the BHXB GX 339-4, which has radio/X-ray coverage during its rise out of quiescence over multiple outbursts, only has (rising) radio/X-ray detections when $L_{\mathrm{X}} \gtrsim 8 \times 10^{34} \mathrm{erg} \mathrm{s}^{-1}$ (3-9 keV; Corbel et al. 2013a, assuming a distance of $\sim 8 \mathrm{kpc}$ ).

There have been (tentative) suggestions that the direction in which a BHXB is moving can influence the normalization of the radio/X-ray correlation, and/or the high-energy radiation mechanisms. For example, for GX 339-4, the radio luminosity could be a factor of $\sim$ two brighter during the hard state decay compared to during the rise (Corbel et al. 2013a). Also, Russell

\footnotetext{
${ }^{20}$ In general, $L_{\mathrm{X}} / L_{\mathrm{Edd}}$ can be used as a rough proxy for $\dot{m}$, but we stress that $L_{\mathrm{X}} / L_{\mathrm{Edd}} \neq \dot{m}$.

${ }^{21}$ An uncooled jet would require less efficient particle acceleration in order to be consistent with the typically soft $\mathrm{X}$-ray spectra $(\Gamma \approx 2)$ of quiescent BHXBs; (e.g., Plotkin et al. 2013; Reynolds et al. 2014).
} 

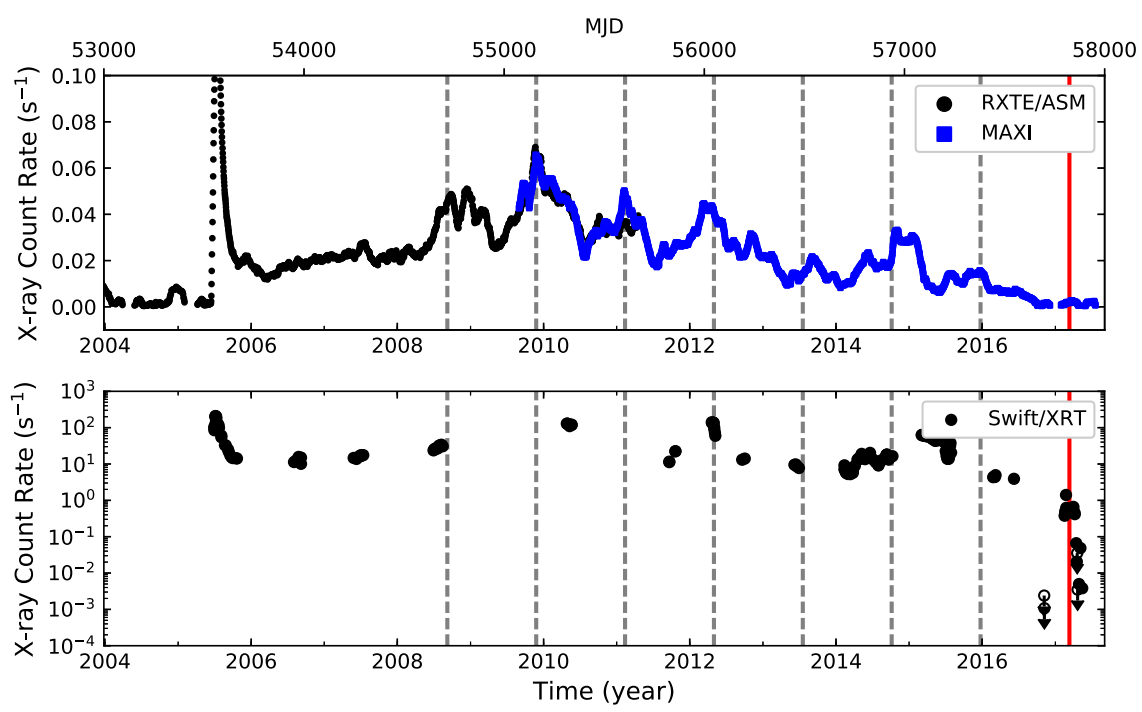

Figure 3. Top panel: RXTE/ASM (black circles) and MAXI (blue squares) X-ray light curves (2-10 keV) toward J1753, showing 30-day running averages from 2004 to 2017. Bottom panel: X-ray light curve from Swift/XRT $(0.3-10 \mathrm{keV})$. The dashed gray lines are separated by 444 days, to mark long-term modulations (anchored to two sharp peaks around MJD $55600=2011$ February 8 and around $56050=2012$ May 3). The final vertical line is shaded red, to highlight that it coincides with the first 2017 mini-outburst.

et al. (2010) find that hard X-rays (3-9 keV) from XTE J1550 -564 could be dominated by synchrotron jet emission during its hard state decay, while jet synchrotron only contributes up to a few percent during the rising hard state. ${ }^{22}$

For J1753, we do not observe a meaningful difference in the radio luminosities (relative to the $\mathrm{X}$-ray) during the rising and decaying hard states. The number of data points is too small to fit for a correlation slope. However, comparing to the radio/Xray correlation for GX 339-4 ( $L_{\mathrm{R}} \propto L_{\mathrm{X}}^{0.62}$; Gallo et al. 2014), J1753 can fall 0.1-0.4 dex below the "standard" track in radio luminosity during the rise and $0.3-0.5$ dex below the "standard" track during the decay, and we have no reason to suspect that the normalization of the radio/X-ray luminosity correlation is systematically different depending on the direction. In the hard state, the jet appears to respond to changes in the inner regions of the accretion flow/jet on short timescales ( $\lesssim 1-2$ days), without a "memory" of whether the jet was brighter or fainter days earlier. Such behavior might be expected: during soft-to-hard state transitions when the jet reactivates after being quenched in the thermal soft state, the radio jet usually appears to turn on at a time that nearly coincides with when the X-ray spectrum again becomes hard and non-thermal, even though it can take 10-30 days for the jet to brighten and become powerful in the infrared waveband as the particle acceleration zone along the jet moves closer to the black hole (Miller-Jones et al. 2012; Corbel et al. 2013b; Kalemci et al. 2013; Russell et al. 2014). The negligible delay between the X-ray and radio wavebands could suggest that the response of the jet to changing amounts of injected power operates on timescales comparable to the time it takes for material to travel outward from the jet base (likely tens of minutes, based on causality arguments and a limited number of $\lesssim 10^{2}$ au size constraints on hard state and quiescent BHXBs, e.g., Dhawan et al. 2000; Stirling et al. 2001; Miller-Jones et al. 2008; Reid et al. 2011, 2014; Russell et al. 2015; Plotkin et al. 2017).

\footnotetext{
${ }^{22}$ Some differences could also be luminosity dependent, as observations of the rising hard state tend to probe higher X-ray luminosities than the decay, due to the hysteretical behavior of BHXB outbursts (Maccarone \& Coppi 2003).
}

\subsection{Long-term Flux Modulations}

During the 2005 outburst of J1753, Shaw et al. (2013) reported X-ray and optical modulations in the long-term light curve with an $\sim 420$ day period. In Figure 3 (upper panel), we produce a light curve of J1753 in the $2-10 \mathrm{keV}$ band over 30day running averages over the entire 11-12 year outburst, using public data from the All-sky Monitor on board the Rossi X-ray Timing Explorer (RXTE/ASM; Levine et al. 1996) and from the Monitor of All-sky X-ray Image (MAXI; Matsuoka et al. 2009). Data with large error bars $\left(>2 \mathrm{cts} \mathrm{s}^{-1}\right.$ for the ASM and $>0.05 \mathrm{cts} \mathrm{s}^{-1}$ for MAXI) were filtered out. ASM and MAXI count rates were divided by factors of 70 and 3 , respectively, to normalize both to Crab units.

Long-term modulations can be seen in Figure 3, which we note are not strictly periodic. The vertical lines in Figure 3 represent the expected peaks of a modulation with a (slightly longer) period of 444 days, which is anchored to the two sharp peaks around MJD 55600 (2011 February 8) and around 56050 (2012 May 3). Most of the vertical lines fall close to local maxima in the light curve. Interestingly, the last vertical line (around MJD $57820=2017$ March 8) falls close to the peak of the first mini-outburst discussed in this work. To better demonstrate this, we show the long-term $0.6-10 \mathrm{keV}$ Swift / XRT light curve in the bottom panel of Figure 3 (this light curve was assembled via the online Swift/XRT data products generator; Evans et al. 2009). The fact that the peak of the first mini-outburst discussed here is close in time to an expected maximum from the long-term modulations suggests that the mini-outbursts are likely still part of the 11-12 year outburst event. This interpretation is supported by optical quiescence not being reached until 2017 July (Zhang et al. 2017).

\subsection{Future Prospects}

Our campaign on J1753 demonstrates the feasibility of obtaining useful radio and X-ray detections in the sparsely sampled low-luminosity regime. The high Galactic latitude of $\mathrm{J} 1753$ is a major reason we are able to obtain X-ray detections at low X-ray luminosities approaching $10^{33} \mathrm{erg} \mathrm{s}^{-1}$ with Swift, 
while even more sensitive X-ray telescopes (e.g., Chandra and $X M M-N e w t o n)$ are required for most other systems. Another reason why the $L_{\mathrm{R}}-L_{\mathrm{X}}$ is not well sampled at low luminosities is that, at these low luminosities, most systems have X-ray fluxes below the detection thresholds of current X-ray all-sky monitors. It is therefore often difficult to trigger more sensitive $\mathrm{X}$-ray observations until the source already has a luminosity above the low-luminosity regime of interest. Our 2017 campaign on J1753 was triggered from changes in its optical flux (via regular monitoring with the Faulkes telescopes; G.-B. Zhang et al. 2017, in preparation), which resulted in VLA and Swift detections at low luminosities. Optical monitoring has previously been shown to be a promising avenue for triggering (and interpreting) multiwavelength observations of BHXBs (e.g., Orosz et al. 1997; Jain et al. 2001a, 2001b; Bernardini et al. 2016; Russell et al. 2017). Our campaign on J1753 further illustrates the utility of optical monitoring to improve coverage of the low-luminosity accretion regime, allowing us to fill in a crucial parameter space to learn about how relativistic jets are coupled to their underlying accretion flows.

We are grateful to the anonymous referee for helpful comments that improved this paper. We thank the VLA for approving our request for DDT observations, and Heidi Medlin and the VLA scientists for their help preparing our observations with a quick turnaround. We thank Brad Cenko for approving the Swift TOO request and the Swift duty scientists for carrying out the observations, and we thank the staff at the Mullard Radio Astronomy Observatory for scheduling and carrying out the AMI-LA observations. We are grateful to Tony Rushton for providing radio and X-ray luminosities for $\mathrm{J} 1753$ in the radiofaint hard state, and to Elena Gallo and Gemma Anderson for helpful discussions. The National Radio Astronomy Observatory is a facility of the National Science Foundation operated under cooperative agreement by Associated Universities, Inc. The AMI telescope is supported by the European Research Council under grant ERC-2012-StG-307215 LODESTONE, and the University of Cambridge. We are grateful for IT knowledge exchange with the SKA project. This work made use of data supplied by the UK Swift Science Data Centre at the University of Leicester. The Faulkes Telescopes are maintained and operated by the Las Cumbres Observatory (LCO). R.M.P. acknowledges support from Curtin University through the Peter Curran Memorial Fellowship. J.C.A.M.J. is supported by an Australian Research Council Future Fellowship (FT140101082). A.W.S. is supported by an NSERC Discovery Grant and a Discovery Accelerator Supplement. T.D.R. acknowledges support from the Netherlands Organisation for Scientific Research (NWO) Veni Fellowship, grant number 639.041.646. A.M.M.S. and T.M.C. gratefully acknowledge support from the European Research Council under grant ERC-2012-StG-307215 LODESTONE. Y.C.P. acknowledges support from a Trinity College JRF.

\section{ORCID iDs}

R. M. Plotkin (1) https://orcid.org/0000-0002-7092-0326 J. C. A. Miller-Jones $\mathbb{~ ( 1 ) ~ h t t p s : / / o r c i d . o r g / 0 0 0 0 - 0 0 0 3 - ~}$ 3124-2814

J. A. Tomsick ㄴ) https://orcid.org/0000-0001-5506-9855

J. Homan $\odot$ https://orcid.org/0000-0001-8371-2713

F. Bernardini (10 https://orcid.org/0000-0001-5326-2010

J. D. Gelfand (i) https://orcid.org/0000-0003-4679-1058

K. J. B. Grainge (1) https://orcid.org/0000-0002-6780-1406
Y. C. Perrott (10 https://orcid.org/0000-0002-6255-8240

A. M. M. Scaife (il https://orcid.org/0000-0002-5364-2301

\section{References}

Al Qasim, A., AlMannaei, A., Russell, D. M., et al. 2017, ATel, 10075, 1 Arnaud, K. A. 1996, in ASP Conf. Ser. 101, Astronomical Data Analysis Software and Systems V, ed. G. H. Jacoby \& J. Barnes (San Francisco, CA: ASP), 17

Bernardini, F., Russell, D. M., Shaw, A. W., et al. 2016, ApJL, 818, L5 Bernardini, F., Zhang, G., Russell, D. M., et al. 2017, ATel, 10325, 1 Blandford, R. D., \& Königl, A. 1979, ApJ, 232, 34

Bright, J., Staley, T., Fender, R., Motta, S., \& Cantwell, T. 2017, ATel, 10110, 1

Burrows, D. N., Hill, J. E., Nousek, J. A., et al. 2005, SSRv, 120, 165

Cadolle Bel, M., Ribó, M., Rodriguez, J., et al. 2007, ApJ, 659, 549

Cao, X.-F., Wu, Q., \& Dong, A.-J. 2014, ApJ, 788, 52

Cash, W. 1979, ApJ, 228, 939

Chen, W., Shrader, C. R., \& Livio, M. 1997, ApJ, 491, 312

Chomiuk, L., Strader, J., Maccarone, T. J., et al. 2013, ApJ, 777, 69

Connors, R. M. T., Markoff, S., Nowak, M. A., et al. 2017, MNRAS, 466,4121

Corbel, S., Aussel, H., Broderick, J. W., et al. 2013b, MNRAS, 431, L107

Corbel, S., Coriat, M., Brocksopp, C., et al. 2013a, MNRAS, 428, 2500

Corbel, S., Fender, R. P., Tomsick, J. A., Tzioumis, A. K., \& Tingay, S. 2004, ApJ, 617, 1272

Corbel, S., Koerding, E., \& Kaaret, P. 2008, MNRAS, 389, 1697

Coriat, M., Corbel, S., Prat, L., et al. 2011, MNRAS, 414, 677

Cseh, D., Webb, N. A., Godet, O., et al. 2015, MNRAS, 446, 3268

Dhawan, V., Mirabel, I. F., \& Rodríguez, L. F. 2000, ApJ, 543, 373

Dong, A.-J., \& Wu, Q. 2015, MNRAS, 453, 3447

Durant, M., Gandhi, P., Shahbaz, T., Peralta, H. H., \& Dhillon, V. S. 2009, MNRAS, 392, 309

Esin, A. A., McClintock, J. E., \& Narayan, R. 1997, ApJ, 489, 865

Evans, P. A., Beardmore, A. P., Page, K. L., et al. 2009, MNRAS, 397, 1177

Falcke, H., Körding, E., \& Markoff, S. 2004, A\&A, 414, 895

Fender, R. P. 2001, MNRAS, 322, 31

Fender, R. P., Gallo, E., \& Jonker, P. G. 2003, MNRAS, 343, L99

Fender, R. P., Maccarone, T. J., \& Heywood, I. 2013, MNRAS, 430, 1538

Froning, C. S., Maccarone, T. J., France, K., et al. 2014, ApJ, 780, 48

Gallo, E., Fender, R. P., Miller-Jones, J. C. A., et al. 2006, MNRAS, 370, 1351

Gallo, E., Migliari, S., Markoff, S., et al. 2007, ApJ, 670, 600

Gallo, E., Miller, B. P., \& Fender, R. 2012, MNRAS, 423, 590

Gallo, E., Miller-Jones, J. C. A., Russell, D. M., et al. 2014, MNRAS, 445, 290

Garcia, M. R., McClintock, J. E., Narayan, R., et al. 2001, ApJL, 553, L47

Gehrels, N., Chincarini, G., Giommi, P., et al. 2004, ApJ, 611, 1005

Greisen, E. W. 2003, in Information Handling in Astronomy-Historical Vistas Vol. 285, ed. A. Heck (Dordrecht: Kluwer), 109

Heinz, S. 2004, MNRAS, 355, 835

Heinz, S., \& Sunyaev, R. A. 2003, MNRAS, 343, L59

Hickish, J., Razavi-Ghods, N., Perrott, Y. C., et al. 2017, arXiv:1707.04237

Jain, R. K., Bailyn, C. D., Orosz, J. A., et al. 2001b, ApJ, 546, 1086

Jain, R. K., Bailyn, C. D., Orosz, J. A., McClintock, J. E., \& Remillard, R. A. 2001a, ApJL, 554, L181

Jonker, P. G., Miller-Jones, J., Homan, J., et al. 2010, MNRAS, 401, 1255

Jonker, P. G., Miller-Jones, J. C. A., Homan, J., et al. 2012, MNRAS, 423, 3308

Kalemci, E., Dinçer, T., Tomsick, J. A., et al. 2013, ApJ, 779, 95

Koliopanos, F., Ciambur, B. C., Graham, A. W., et al. 2017, A\&A, 601, A20

Kong, A. K. H. 2017, ATel, 10081, 1

Körding, E. G., Fender, R. P., \& Migliari, S. 2006, MNRAS, 369, 1451

Kraft, R. P., Burrows, D. N., \& Nousek, J. A. 1991, ApJ, 374, 344

Levine, A. M., Bradt, H., Cui, W., et al. 1996, ApJL, 469, L33

Lewis, F., Roche, P., Russell, D. M., \& Fender, R. P. 2008, in AIP Conf. Ser. 1010, A Population Explosion: The Nature Evolution of X-ray Binaries in Diverse Environments, ed. R. M. Bandyopadhyay et al. (New York: AIP), 204

Maccarone, T. J. 2005, MNRAS, 360, L30

Maccarone, T. J., \& Coppi, P. S. 2003, MNRAS, 338, 189

Markoff, S., Nowak, M., Corbel, S., Fender, R., \& Falcke, H. 2003, A\&A, 397,645

Matsuoka, M., Kawasaki, K., Ueno, S., et al. 2009, PASJ, 61, 999

McClintock, J. E., Narayan, R., Garcia, M. R., et al. 2003, ApJ, 593, 435

McMullin, J. P., Waters, B., Schiebel, D., Young, W., \& Golap, K. 2007, in ASP Conf. Ser. 376, Astronomical Data Analysis Software and Systems XVI, ed. R. A. Shaw, F. Hill, \& D. J. Bell (San Francisco, CA: ASP), 127 
Merloni, A., Heinz, S., \& di Matteo, T. 2003, MNRAS, 345, 1057

Mezcua, M. 2017, IJMPD, 26, 1730021

Miller, B. P., Gallo, E., Greene, J. E., et al. 2015, ApJ, 799, 98

Miller-Jones, J. C. A., Gallo, E., Rupen, M. P., et al. 2008, MNRAS, 388, 1751

Miller-Jones, J. C. A., Jonker, P. G., Maccarone, T. J., Nelemans, G., \& Calvelo, D. E. 2011, ApJL, 739, L18

Miller-Jones, J. C. A., Strader, J., Heinke, C. O., et al. 2015, MNRAS, 453, 3918

Miller-Jones, J. C. A., Wrobel, J. M., Sivakoff, G. R., et al. 2012, ApJL, 755, L1

Orosz, J. A., Remillard, R. A., Bailyn, C. D., \& McClintock, J. E. 1997, ApJL, 478, L83

Palmer, D. M., Barthelmey, S. D., Cummings, J. R., et al. 2005, ATel, 546, 1

Perley, R. A., \& Butler, B. J. 2013, ApJS, 204, 19

Plotkin, R. M., Gallo, E., \& Jonker, P. G. 2013, ApJ, 773, 59

Plotkin, R. M., Gallo, E., Markoff, S., et al. 2015, MNRAS, 446, 4098

Plotkin, R. M., Markoff, S., Kelly, B. C., Körding, E., \& Anderson, S. F. 2012, MNRAS, 419, 267

Plotkin, R. M., Miller-Jones, J. C. A., Gallo, E., et al. 2017, ApJ, 834, 104

Plotkin, R. M., Miller-Jones, J. C. A., Jonker, P. G., et al. 2016, ATel, 9765, 1

Qiao, E., \& Liu, B. F. 2013, ApJ, 764, 2

Ratti, E. M., Jonker, P. G., Miller-Jones, J. C. A., et al. 2012, MNRAS, 423, 2656

Reid, M. J., McClintock, J. E., Narayan, R., et al. 2011, ApJ, 742, 83

Reid, M. J., McClintock, J. E., Steiner, J. F., et al. 2014, ApJ, 796, 2

Remillard, R. A., \& McClintock, J. E. 2006, ARA\&A, 44, 49

Reynolds, M. T., Reis, R. C., Miller, J. M., Cackett, E. M., \& Degenaar, N. 2014, MNRAS, 441, 3656

Ribó, M., Munar-Adrover, P., Paredes, J. M., et al. 2017, ApJL, 835, L33

Rodriguez, J., Cadolle Bel, M., Tomsick, J. A., et al. 2007, ApJL, 655, L97

Rushton, A. P., Shaw, A. W., Fender, R. P., et al. 2016, MNRAS, 463, 628

Russell, D. M., Al Mannaei, A., Al Qasim, A., et al. 2016, ATel, 9708, 1

Russell, D. M., Al Qasim, A., Bernardini, F., et al. 2017, ApJ, submitted (arXiv:1707.05814)

Russell, D. M., Maitra, D., Dunn, R. J. H., \& Markoff, S. 2010, MNRAS, 405,1759
Russell, T. D., Miller-Jones, J. C. A., Curran, P. A., et al. 2015, MNRAS, 450, 1745

Russell, T. D., Soria, R., Miller-Jones, J. C. A., et al. 2014, MNRAS, 439, 1390

Shaw, A. W., Charles, P. A., Bird, A. J., et al. 2013, MNRAS, 433, 740

Shaw, A. W., Charles, P. A., Casares, J., \& Hernández Santisteban, J. V. 2016a, MNRAS, 463, 1314

Shaw, A. W., Tomsick, J. A., Bahramian, A., et al. 2016b, ATel, 9735, 1

Shaw, A. W., Tomsick, J. A., Zhang, G., et al. 2017, ATel, 10288, 1

Sobolewska, M. A., Papadakis, I. E., Done, C., \& Malzac, J. 2011, MNRAS, 417,280

Soleri, P., \& Fender, R. 2011, MNRAS, 413, 2269

Soleri, P., Fender, R., Tudose, V., et al. 2010, MNRAS, 406, 1471

Stirling, A. M., Spencer, R. E., de la Force, C. J., et al. 2001, MNRAS, 327,1273

Strader, J., Chomiuk, L., Maccarone, T. J., Miller-Jones, J. C. A., \& Seth, A. C. 2012, Natur, 490, 71

Swinbank, J. D., Staley, T. D., Molenaar, G. J., et al. 2015, A\&C, 11, 25

Tetarenko, B. E., Bahramian, A., Arnason, R. M., et al. 2016, ApJ, 825, 10

Tomsick, J. A., Rahoui, F., Kolehmainen, M., et al. 2015, ApJ, 808, 85

Tomsick, J. A., Shaw, A., Gandhi, P., Rahoui, F., \& Dincer, T. 2017, ATel, 10114, 1

Verner, D. A., Ferland, G. J., Korista, K. T., \& Yakovlev, D. G. 1996, ApJ, 465,487

Wilms, J., Allen, A., \& McCray, R. 2000, ApJ, 542, 914

Xie, F.-G., \& Yuan, F. 2017, ApJ, 836, 104

Xue, Y. Q., \& Cui, W. 2007, A\&A, 466, 1053

Yuan, F., \& Cui, W. 2005, ApJ, 629, 408

Yuan, F., \& Narayan, R. 2014, ARA\&A, 52, 529

Yuan, F., Yu, Z., \& Ho, L. C. 2009, ApJ, 703, 1034

Zdziarski, A. A., Gierliński, M., Mikołajewska, J., et al. 2004, MNRAS, 351, 791

Zhang, G., Russell, D. M., Bernardini, F., Gelfand, J. D., \& Lewis, F. 2017, ATel, 10562, 1

Zwart, J. T. L., Barker, R. W., Biddulph, P., et al. 2008, MNRAS, 391, 1545 